\title{
ASPECTS OF THE DYNAMICS OF PNEUMATIC SWITCHES
}

\author{
DRAGOMIRESCU, C[ristian] G[eorge] \& ILIESCU, V[ictor]
}

\begin{abstract}
The paper is proposing a study model for the motion of the rod if a pneumatic switch. It is also offering a model for the response analysis of the electro valve of the pneumatic switch in the case of a perturbation similar to one that may occur in real use. For certain values of the coefficients of the dynamic equations, some variations in time as well as the phase plane diagrams are presented in order to point out the dynamic characteristics of the studied system with the aim to better predict the possible motions of the studied system and, possibly, to a better design the pneumatic switch
\end{abstract}

Key words: pneumatic switch, electro valve, perturbation, vibration

\section{INTRODUCTION}

The pneumatic (air compressed) switches are used in the technical applications requiring a relatively large number of commutations and the quick regain of the dielectric stiffness after a shut down (electric furnaces, electric circuits of $24 \mathrm{kV}$ and 4000A condensers batteries, high power electric motors etc.). The compressed air is used to move the mobile element and, in the same time, to extinguish the voltaic arc produced during the motion (Deciu et al., 2002).

Regardless the constructive shape, the principle is the same: after a certain number of actions, the pressure is reaching its minimum value and an electro valve permits the air access into the reservoir in order to increase the pressure to its maximal value (Fig. 1 (a))

In Fig. 1 (b) is shown the model of the contact of a pneumatic switch. The compressed air is moving the rod compressing the elastic element, in order to aloud the electric contact. At low pressure, the elastic element is moving the rod up and is switching off the contact. The motion is damped (using also compressed air) in order to avoid the hard collision of the rod with the body of the switch. The largest part of the bracketing energy is transformed in thermal energy.

\section{THE MODELS AND THE STUDY}

The motion equation is (Deciu et al., 2001), (Deciu et al., 2002), (Radeş, 2009):

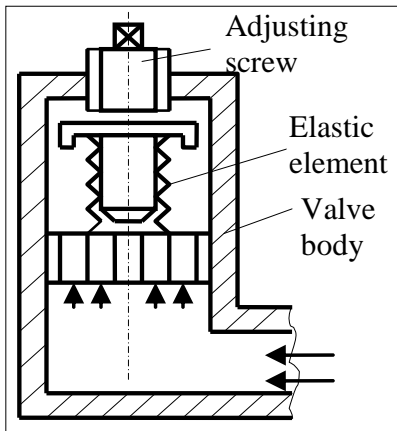

(a) The electro valve

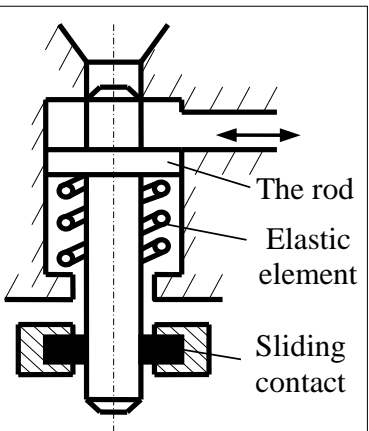

(b) The pneumatic switch
Fig. 1. The electro valve and the pneumatic switch

$$
M \frac{d^{2} y}{d t^{2}}+c\left(y_{0}+y\right)=p_{0} A+\alpha A t+M g
$$

where $M$ is the rod mass, $c$ - the elasticity coefficient, $y_{0}$ - the initial deformation of the spring, $p_{0}$ - pressure needed for the motion to start $\left(p_{0} A \approx c y_{0}\right), \alpha$ - pressure increasing coefficient $\left(N / m^{2} s\right), A-$ the active surface of the piston, $g-$ the gravitational acceleration.

Out of the equation (1), the response $y$ and the speed $v$ are:

$$
\begin{aligned}
& y=\frac{C_{1}}{\omega^{3}}(\omega t-\sin \omega t)+\frac{C_{2}}{\omega^{2}}(1-\cos \omega t) \\
& v=\frac{C_{1}}{\omega^{2}}(1-\cos \omega t)+\frac{C_{2}}{\omega} \sin \omega t
\end{aligned}
$$

where $C_{1}=\frac{\alpha A}{M}, \omega=\sqrt{\frac{c}{M}}$ and $C_{2}=\frac{p_{0} A-c y_{0}-\mu N+M g}{M}$.

For $M=0.25 \mathrm{Kg}, c=2500 \mathrm{~N} / \mathrm{m}$ one may obtain the following time variation for motion (2) and speed (3) (Fig. 2).

In order to go further, we used the phase plane diagrams because they are offering more information about the system dynamic. One may observe that for different time values $(t=$ $50 \mathrm{~s}, 20 \mathrm{~s}, 5 \mathrm{~s}$ and $0.25 \mathrm{~s}$, respectively) a closed loop is obtained (Fig. 3), pointing out a periodical motion (Voinea et al., 2000).

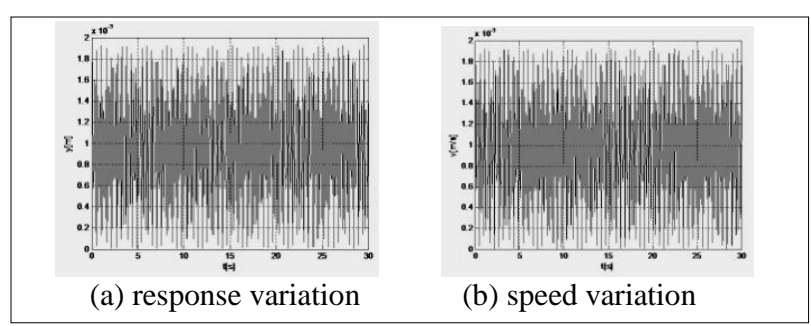

Fig. 2. Displacement and speed variation in time

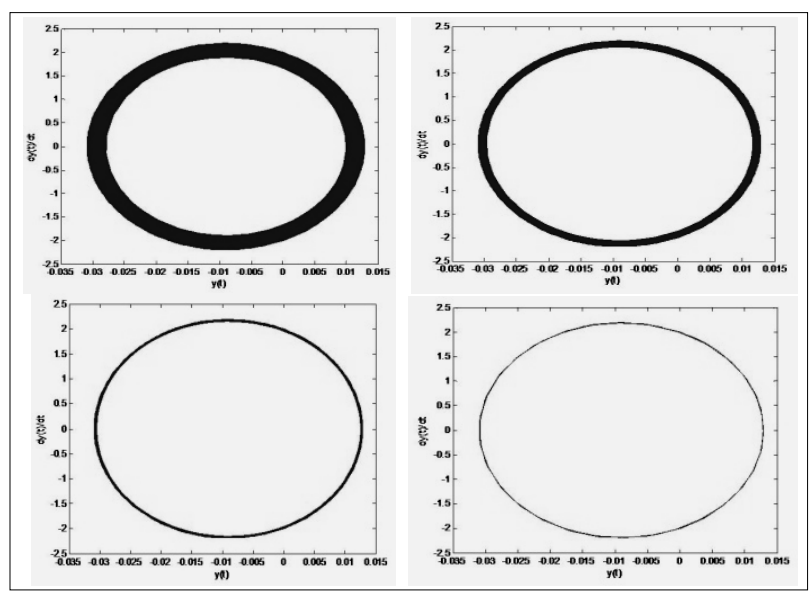

Fig. 3. The phase plane diagrams 


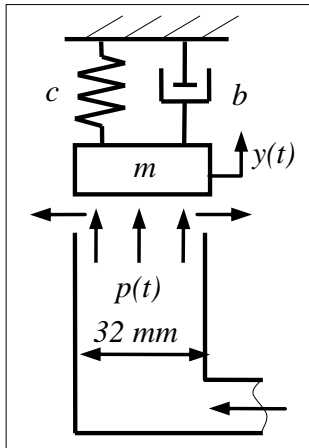

(a) The study model

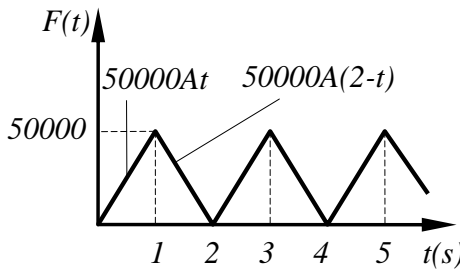

(b) The periodical force
Fig. 4. The study model and the force applied

If one is analysing the function $y(t)$ in the case of a distributed force (due to the pressure of the air) and of a damped motion (due to the frictions), one may obtain the model in Fig 4 (a).

For the numerical study we considered the diameter $d=$ $32 \mathrm{~mm}$, the pressure $p_{0}=20 \mathrm{bar}$, the force inducing the perturbation $F_{p}(t)=F(t)-\frac{\pi d^{2}}{4} \cdot p(t)$. Assuming that $F(t)$ is periodical having the period 2 s (Fig. 4 (b)), and using the Fourier function (Deciu et al., 2001), (Gross et al., 2010):

$$
F(t)=\frac{a_{0}}{2}+a_{1} \cos \omega t+a_{2} \cos 2 \omega t+\ldots+b_{1} \sin \omega t+b_{2} \sin 2 \omega t+\ldots
$$

where $a_{j}=\frac{2}{\tau} \int_{0}^{2} F(t) \cos (j \omega t) d t, b_{j}=\frac{2}{\tau} \int_{0}^{2} F(t) \sin (j \omega t) d t,(\mathrm{j}=0,1,2, \ldots)$, one may obtain the Fourier coefficients (Tab. 1).

Considering only the first three vibration modes we approximated the force as follows:

$$
F(t)=25000 A-\frac{2 \cdot 10^{5}}{\pi^{2}} A \cos (\omega \cdot t)-\frac{2 \cdot 10^{5}}{9 \pi^{2}} A \cos (3 \omega \cdot t)
$$

This lead to the stationary response of the perturbed electro valve:

$$
\begin{gathered}
y=\frac{25000}{c} A-\frac{\frac{2 \cdot 10^{5}}{c \pi^{2}} A}{\sqrt{\left(1-\tau^{2}\right)^{2}+(2 \zeta \tau)^{2}}} \cos \left(\omega \cdot t-\phi_{1}\right)- \\
-\frac{\frac{2 \cdot 10^{5}}{9 c \pi^{2}} A}{\sqrt{\left(1-9 \tau^{2}\right)^{2}+(6 \zeta \tau)^{2}}} \cos \left(3 \omega \cdot t-\phi_{3}\right)
\end{gathered} .
$$

The angular frequencies of the electro valve, $\omega_{0}$, and of the perturbation, $\omega$, are:

$$
\omega_{0}=\sqrt{\frac{c}{M}}=\sqrt{\frac{2500}{0.25}}=100 \mathrm{rad} / \mathrm{s}, \omega=\frac{2 \pi}{\tau}=\frac{2 \pi}{2}=\pi \mathrm{rad} / \mathrm{s} .
$$

With these values we calculated the ratio of the angular frequencies, $r$, the ratio of the damper, $\zeta$ :

$$
r=\frac{\omega}{\omega_{0}}=0.031416, \zeta=\frac{c}{c_{0}}=\frac{c}{2 m \omega_{0}}=\frac{10.0}{2 \cdot 0.25 \cdot 100}=0.2 .
$$

\begin{tabular}{|c|c|c|c|c|c|}
\hline$j$ & 0 & 1 & 2 & 3 & $4 \ldots$ \\
\hline$a_{j}$ & $50000 A$ & $-2 \cdot 10^{5} \cdot A / \pi^{2}$ & 0 & $-2 \cdot 10^{5} \cdot A / 9 \pi^{2}$ & 0 \\
\hline$b_{j}$ & - & 0 & 0 & 0 & 0 \\
\hline
\end{tabular}

Tab. 1. The Fourier coefficients

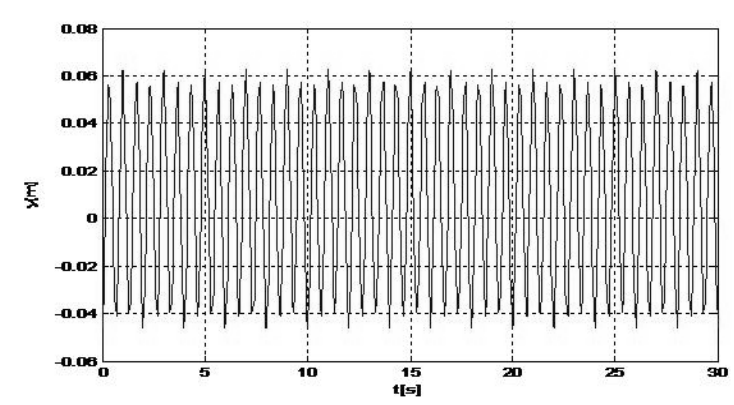

Fig. 5. Time variation of the electro valve response

With the found values for $r$ and $\zeta$, we then calculated the phases $\phi_{1}, \phi_{3}$ :

$$
\left.\begin{array}{l}
\phi_{1}=\arctan \frac{2 \zeta r}{1-r^{2}}=\arctan \frac{2 \cdot 0.2 \cdot 0.031416}{1-0.031416}=0.0125664 \mathrm{rad} \\
\phi_{3}=\arctan \frac{6 \zeta r}{1-9 r^{2}}=\arctan \frac{6 \cdot 0.2 \cdot 0.031416}{1-9 \cdot 0.031416}=0.0380483 \mathrm{rad}
\end{array}\right\}
$$

Now was possible to finally calculate the response $y(t)$ of the electro valve:

$$
\begin{aligned}
y(t)=0.0080425- & 0.00210 \cos (\pi \cdot t-0.0125664)- \\
- & 0.05241136 \cos (3 \pi \cdot t-0.0380483)
\end{aligned}
$$

and to represent its variation in time (Fig. 5). It is easy to observe the periodic motion.

\section{CONCLUSIONS}

The paper is proposing a study model in the case of a periodic perturbation, using the dynamic systems theory as a new approach, considering the time evolution of a significant variable, the phase plane diagrams etc.

The model used was considering certain elasticity as well as a damper, according to the real material characteristics. The coefficients were subsequently deduced.

The numerical simulations were done using coefficients values taken from the speciality books (Deciu et al., 2002).

In studied cases, the permanent/stationary working stage was considered (natural vibration of the system has stopped due to the damper). This assumption is coherent with the usual technical approach. For the first mode of the perturbation and for the considered conditions, a periodical response of the perturbed electro valve was obtained (Fig. 5).

Further studies will be carried out considering different types of perturbations, more numerical values to cover a wider area and taking into account the nonlinearities of the system.

\section{REFERENCES}

Deciu, E.; Dragomirescu, C. (2001). Maschinendynamik, Editura PRINTECH, ISBN 973-652-438-8, Bucureşti

Deciu, E.; Bugaru, M.; Dragomirescu, C. (2002). Vibratii neliniare $\mathrm{cu}$ aplicaţii $\hat{\imath}$ ingineria mecanică, Editura Academiei Române, ISBN 973-27-0911-1, Bucureşti

Gross, D.; Hauger,W.; Schröder, J.; Wall, W. (2010). Technische Mechanik 3, Springer Verlag Heidelberg Dordrecht London New York, Printing and Binding:Stürtzt GmbH, ISBN 978-3-642-11263-8, e-ISBN 978-3-64211264-5, Würzburg

Voinea, R.; Stroe, I. (2000). Introducere in teoria sistemelor dinamice, Editura Academiei Române, ISBN 973-27-07399, Bucureşti

Radeş, Mircea (2009). Dynamics of Machinery, Editura PRINTECH, ISBN 978-606-521-342-5, Bucureşti 